\title{
DIDÁCTICA Y VIVENCIAS DEL SERVICIO DE APOYO DE LA ESCUELA JOSÉ RAMÓN HERNÁNDEZ
}

\author{
Angélica Fontana Hernández ${ }^{1}$
}

\section{Resumen}

La atención a la diversidad es un reto que enfrenta el sistema educativo costarricense y las transformaciones que conlleva empujan desde sus bases poco a poco los cambios requeridos.

El reconocimiento de la diversidad estudiantil en el ámbito escolar es un hecho ineludible, porque cada estudiante coexiste en distintas realidades, estructuras familiares, contextos sociales y culturales donde las diferencias y semejanzas juegan un papel determinante en la convivencia humana.

Los servicios de apoyo están inmersos en esta realidad. La población estudiantil que presenta necesidades educativas derivadas de una discapacidad o de una condición especial, demanda cada vez más diversificar los espacios de intervención pedagógica empleando estrategias innovadoras y adaptadas a sus ca- racterísticas y necesidades. Asimismo, que les permita un aprendizaje significativo y un desarrollo integral.

\section{Abstract}

At the present time, Costa Rican educational system faces the challenge to deal with diversity while the necessary transformations are emerging gradually to achieve the required changes.

Recognition of student's diversity within the educational environment is an irrefutable fact because each student coexists in different realities, family structures, and social and cultural contexts, where differences and similarities play an important role in the human life.

Supporting services are immersed in this reality. Student population with educational needs derived from disability or special conditions, demands diversification of pedagogic intervention spaces

\footnotetext{
'Bachiller en Problemas de Aprendizaje, Licenciada en Educación Especial con énfasis en Retardo Mental y Máster en Pedagogía con énfasis en la diversidad de procesos educativos de la Universidad Nacional. Académica e investigadora de la División de Educación Básica del CIDE. Tiene experiencia en la ecuación primaria y educación especial y tiene varias publicaciones en el campo de la pedagogía.
} 
by means of innovative and individually adapted strategies. This will allow significant leaming and integral development.
Palabras claves: Educación especial, servicios de apoyo, experiencia en el aula.

C

ada mañana un grupo de niños y niñas se acercan al aula, curiosean con sus ojos brillantes, saludan y preguntan:

“-¿Cuándo me toca venir, Niña?

- Vamos a ver el horario, a Jéssica le toca venir el viernes a las 8:30 de la mañana y a usted, Ángel, le corresponde el martes a las 10:00 de la mañana.

-iQué dicha! Me voy porque tocaron el timbre -dice Ángel y sale presuroso.

Jéssica se aleja en forma silenciosa y con una sonrisa en sus labios."

Cuando el ambiente del aula escolar es agradable y acogedor los y las estudiantes siempre muestran interés por aprender y asistir puntualmente. Son muchos los factores que inciden en su motivación, pero uno de los más importantes es la interacción docente/discente. Cuando el o la estudiante siente que es apreciado y aceptado por el o la docente, que puede ser amigo de sus compañeros y compañeras y que es capaz de aprender, incluso aquellos temas difíciles, mostrará una actitud asertiva en los procesos de enseñanza y aprendizaje. El buen trato, el intercambio de afecto, la tolerancia, el respeto y la confianza inquebrantable de ser mejor cada día provocarán en su interior, la voluntad suficiente para superarse y realizar el esfuerzo necesario para aprender.

Con estudiantes que presentan necesidades educativas derivadas de una discapacidad o de una condición especial, hay que ser muy cuidadoso en la selección y aplicación de las estrategias de enseñanza. El presente artículo retoma algunas vivencias y técnicas didácticas que se utilizan en el Servicio de Apoyo de la escuela José Ramón Hernández Badilla, con el fin de socializar la experiencia docente y de generar un proceso de intercambio y enriquecimiento de la labor que se realiza en los servicios de Educación Especial.

El servicio de apoyo inicia su labor con un espacio preinstruccional, en el cual, los y las estudiantes comparten las vivencias del día anterior y se expresan las inquietudes, dudas, sentimientos y emociones del día presente. Por lo general, en este período de círculo, se siguen rutinas, tales como el repaso de los hábitos de higiene y de trabajo escolar, el cuidado personal, los 
comportamientos esperados, el estado del tiempo, celebración de acontecimientos importantes (cumpleaños de estudiantes), entre otras actividades.

La habilidad del docente consiste en orientar y preparar a los discentes para el aprendizaje de nuevos temas o el seguimiento de otros ya comenzados. Es en este momento, cuando se les indica a los y las estudiantes cuál es el propósito de las actividades que se realizarán en el área de Español o Matemáticas. Casi siempre expresan su conformidad e inconformidad, se miran entre ellos, hacen gestos o muecas de aceptación o rechazo y los más extrovertidos dicen:

“-¡Otra vez Matemáticas!

- ¡Trabajemos en Español!

- ¡Sí, Niña! -dicen todos en voz alta.

-¡Está bien! Cada uno saque su libro de lectura."

Si empleamos la estrategia de organizados previos, estos no deben ser muy largos, se puede utilizar oraciones o párrafos cortos que ayuden a los estudiantes a ubicar los conceptos centrales de los temas que se van a estudiar.

En fase de coinstrucción, el Servicio de Apoyo intenta mantener las condiciones básicas para desplegar en los discentes nuevas habilidades académicas, lingüísticas, emocionales, sociales y espirituales. Por lo tanto, se retoman de los siguientes principios metodológicos: la flexibilidad, la adecuación, la intuición, la internalización, la reflexión y la transferencia $(\mathrm{Mu}-$ ñoz, A. y Noriega, J., 1996) en los procesos de lectura, escritura y lógicomatemáticos básicos.

La enseñanza de la lengua escrita es un tema central en los Servicios de Apoyo, porque los discentes en su mayoría presentan dificultades para leer y escribir en forma independiente y han tenido experiencias negativas con al menos uno o dos métodos de lectura (Método Fonético, Silábico, de palabra clave, de oraciones, el Ecléctico, el Natural Integral, entre otros).

En el Servicio de Apoyo, empleamos una concepción constructivista en la elaboración de textos, con el fin de desplegar la capacidad linguiística y de recuperar lo aprendido por los discentes anteriormente. Entre las estrategias usadas, se pueden mencionar:

1. Creación de palabras y oraciones en forma grupal e individual, con el vocabulario conocido, apoyo gráfico y fonético.

2. Construcción guiada de textos cortos y realización de ejercicios de comprensión de lectura con apoyo docente, de un compañero o compañera. 
3. Construcción independiente de textos y realización de variados ejercicios de comprensión de lectura.

4. Elaboración guiada de un libro de lectura y escritura.

Cada estudiante puede trabajar en forma individual en su libro de lectura. Unos están construyendo un texto corto con el vocabulario conocido, otros lo ilustran con variedad de dibujos y colores, y algunos, contestan preguntas de comprensión de lectura de los textos elaborados por ellos mismos. Es fundamental respetar el ritmo y estilo de aprendizaje de cada discente y mantener un seguimiento personal de las creaciones, reconstrucciones y lectura de los textos elaborados.

Si queremos enriquecer los procesos de lectura y escritura, podemos emplear variadas estrategias, tales como el análisis fonético o silábico de las palabras, apoyos gráficos, como dibujos de historietas, cuentos y anécdotas según lo requiera, el discente o el grupo de estudiantes. En ocasiones, se construyen carteles de experiencias en forma grupal para incorporarlos en los libros personales y otras veces se comparten los trabajos elaborados en forma individual.

El proceso de coinstrucción en el área de Español dura, aproximadamente, dos a cuatro lecciones máximo, luego, es conveniente cambiar de materia. En este momento, los discentes están un poco cansados por el esfuerzo realizado y el juego libre o dirigido prepara a las y los estudiantes para las actividades siguientes. Por esta razón, se realizan diversos juegos de cálculo mental (sumas - restas - multiplicaciones), de resolución de problemas simples en forma oral, adivinanzas, formación de secuencias ascendentes y descendentes, el número antecesor y el sucesor, entre otros.

Cada estudiante trabaja en forma individual, con diversos materiales concretos (paletas, tucos, bloques multibase, números y otros) y gráficos, que le permitan construir los conceptos básicos, resolver los ejercicios asignados, poner en práctica los conocimientos adquiridos y desarrollar mayores destrezas matemáticas. También, se trabaja en grupo, específicamente, para compartir las estrategias personales en la resolución de los problemas matemáticos y el repaso del algoritmo de las operaciones fundamentales.

Además, coordinamos con los docentes de I y II ciclos para determinar los contenidos de Ciencias y Estudios Sociales que requieren reforzarse con los discentes que asisten al servicio de Apoyo. Con el propósito de que se logren mayores aprendizajes en estas materias, empleamos variadas estrategias de enseñanza, siendo la explicación y la exposición con apoyo gráfico las 
Cuando el ambiente del aula escolar es agradable y acogedor los y las estudiantes siempre muestran interés por aprender y asistir puntualmente. Son muchos los factores que inciden en su motivación, pero uno de los más importantes es la interacción docentel discente. Cuando el o la estudiante siente que es apreciado y aceptado por el o la docente, que puede ser amigo de sus compañeros y compañeras y que es capaz de aprender, incluso aquellos temas dificiles, mostrará una actitud asertiva en los procesos de enseñanza y aprendizaje. técnicas más utilizadas. Las ilustraciones empleadas, por lo general, son descriptivas, expresivas y funcionales, porque facilitan la comprensión de los temas. Las técnicas de construcción de resúmenes escritos, redes semánticas y los mapas conceptuales son muy complejas para aplicarlas con los discentes que presentan necesidades educativas, ya que requieren de un período largo de entrenamiento y de habilidades específicas, tales como análisis, síntesis, memoria, entre otras.

En la fase de posintruccional, realizamos diversas estrategias que permitan a los discentes repasar los aspectos más importantes abordados en la clase. Entre las técnicas más utilizadas se pueden mencionar: resúmenes orales, formulación de preguntas, elaboración de dibujos, esquemas, collage, murales y tareas cortas para que pongan en práctica

lo aprendido. En los últimos momentos. hacemos diversos ejercicios de relajación o escuchamos música.

Para procurar un equilibrio entre el tiempo dedicado a cada disciplina básica en el Servicio de Apoyo, un día se inicia con Español y se continúa con Matemáticas y el día siguiente a la inversa. No obstante, los estudiantes tienen sus preferencias; algunos les gusta trabajar más en lectura y escritura y otros en sumas y restas o problemas matemáticos. Se pretende considerar los intereses de los discentes y de atender sus necesidades. En ocasiones se sugiere una estrategia y en otras se les permite que ellos seleccionen los temas y las actividades que desean realizar.

Por las características y necesidades educativas que presentan los estudiantes que atiende el Servicio de Apoyo. el docente tiene libertad para seleccionar el tipo de currículo y las estrategias de enseñanza que considere más apropiado. Esta autonomía profesional le permite al docente de Educación 
Especial desplegar su capacidad creadora, implementando técnicas innovadoras y el juego como una estrategia eficaz para el aprendizaje significativo.

Si reflexionamos sobre los principios metodológicos mencionados anteriormente, se puede afirmar que la flexibilidad, la adecuación, la intuición y la internalización están presentes en las diversas estrategias de enseñanza utilizadas en el Servicio de Apoyo. No obstante, los principios de reflexibilidad y transferencias son más difíciles de lograr, porque requieren procesos cognitivos complejos, tales como memoria (inmediata, mediata y a largo plazo), interrelaciones entre las ideas, integración de los conocimientos adquiridos, habilidades de observación, análisis, síntesis y transferencia (Muñoz, A. y Noriega, J., 1996, 107-108), en las cuales los discentes presentan serias dificultades. Por lo tanto, se necesita de un período prolongado de entrenamiento con variados ejercicios y que tenga diferentes niveles de aproximación.

En ocasiones, creemos que los discentes con necesidades educativas son pocos creativos, pero de acuerdo con la experiencia del Servicio de Apoyo, esta capacidad es la que más se percibe si se proporciona la mediación pedagógica adecuada y los momentos significativos.

Cada estudiante tiene su propio estilo y ritmo de aprendizaje, su temperamento, sus calidades y habilidades, sus necesidades educativas, su contexto familiar, escolar y comunal que determinan la "disciplina personal de trabajo" (Sánchez, M. et al. 2001 p. 51). Esta se refleja en los trabajos escolares cotidianos, pero tiene su mayor expresión en los momentos de recreación y juego, porque los estudiantes son capaces de crear música con sonidos e instrumentos diferentes, de pintar con las combinaciones más extravagantes, de construir murales con recursos naturales y artificiales, de expresar figuras literarias (prosopopeyas/símiles/hipérboles) con un vocabulario sencillo, de realizar dramatizaciones y de narrar cuentos, historietas y anécdotas insólitas.

Si consideramos que las estrategias de enseñanza que imparte el docente deben convertirse en una herramienta eficaz para el aprendizaje de sus discentes, podemos afirmar que el Servicio de Apoyo intenta mantener una relación sincrónica entre la intervención pedagógica y el proceso constructivo de los conocimientos por parte de los y las estudiantes. Para logralo, se realizan diferentes acciones, entre ellas se pueden mencionar:

1. Se efectúa un diagnóstico pedagógico a cada estudiante, en el cual se identifican los niveles de conocimiento que posee en las materias de Matemáticas y Español y se determinan los puntos significativos de partida de cada conocimiento básico. 
2. Se seleccionan las estrategias de enseñanza, de acuerdo con las características y necesidades de los discentes. Además, se proporciona diferentes tipos de soportes y recursos de apoyo.

3. Se trabajan diferentes técnicas a nivel emocional para motivar el esfuerzo personal ante las múltiples situaciones que se les presenta en el contexto escolar.

Por otra parte, las estrategias de enseñanza utilizadas en el Servicio de Apoyo, intentan favorecer la creación de zonas de desarrollo próximo (ZDP) en la construcción de los conocimientos y de las habilidades por parte de los discentes. Para ello, se realiza un análisis de tareas del trabajo que se debe hacer, especificando los puntos de aproximación (procesos) requeridos para completar una tarea con ayuda o en forma independiente. Durante este período de construcción individual, los y las estudiantes emplean diferentes formas para entender o enfrentar el trabajo asignado y, en ocasiones, encuentran rutas más cortas a las planeadas.

Un ejemplo que ilustra este proceso de creación de ZDP fue el vivido con Jessica el año pasado. La estudiante había repetido tres veces segundo grado y tenía 12 años de edad. Cuando ingresó al Servicio de Apoyo se reubicó en un tercer grado con una adecuación significativa. Presentaba dificultades para leer y escribir, se ausentaba sin justificación y mostraba desinterés por el estudio.

Se inició el proceso de lectura y escritura con el Método Ecléctico, específicamente, con la estrategia metodológica de Textos elaborados por los niños y niñas. Formó las primeras palabras y oraciones con apoyo gráfico y las ilustró. Luego, con ayuda de una secuencia de dibujos escribió el primer texto. Las oraciones eran cortas y con repetición de palabras. Sin embargo, cada día mostraba más interés por leer y escribir sus propios textos y realizar variados ejercicios de comprensión de lectura en forma oral y escrita. Este proceso de construcción y de análisis de texto fue creciendo rápidamente, hasta que un día conversando con la estudiante descubrimos cuál era su dificultad específica.

"-Jessica ¿Te gusta leer y escribir?

- ¡Sí Niña! Pero me cuesta leer y escribir algunas palabras con bra / bla (haciendo un esfuerzo con la lengua). Las confundo y escribo mal.

-Con práctica y esfuerzo vamos a mejorar la lectura y escritura de esas sílabas y muchas más." 
Luego, se realizaron variados ejercicios para mejorar la pronunciación de las letras r y l, se le asignó una clave de color a cada sílaba (rojo para la sílaba bra y azul para la sílaba bla) que se emplearía para la discriminación auditiva en los ejercicios de lectura oral y para la discriminación visual en los ejercicios de compresión escrita. Desde este momento, sus avances en la elaboración de textos fueron significativos y continúa alcanzando progresos.

De acuerdo con las observaciones realizadas en las clases de materias básicas de los discentes del Servicio de Apoyo, se ha podido percibir que las estrategias de enseñanza empleadas por los docentes de I y II ciclos responden más a la secuencia lógica de un método específico o de una disciplina específica, que a las características y necesidades de los alumnos y las alumnas. Situación lamentable, porque no permite la creación de ZDP en la construcción de los conocimientos

Si bien es cierto, que las estrategias de enseñanza aplicadas en el Servicio de Apoyo son diversas y adaptadas a las características y necesidades de los discentes, consideramos que las mismas no constituyen un desafío a la comprensión y actuación de los y las estudiantes. Más bien, en reiteradas ocasiones, se apegan tanto a las exigencias del currículo y a la adquisición de las habilidades básicas que pasan inadvertido los talentos de los estudiantes en las distintas disciplinas (canto, baile, oratoria, dibujos, expresión corporal). Muestra de esta situación, es que la mayoría de los docentes de Educación Especial y de I y II ciclos no planifican en forma intencionada actividades curriculares y extracurriculares que favorezca el desarrollo de talentos; y las pocas veces en que se realizan Ferias Científicas, concursos de oratoria o de dibujo responden más a las exigencias de la Dirección Regional, de la Dirección de la institución educativa o de un Comité específico, que al fortalecimiento de la diversidad personal.

Para concluir, es fundamental que los docentes diversifiquen los espacios de intervención profesional empleando como estrategia la autorefiexión de su acción pedagógica y, de esta forma, llevar a la práctica los conocimientos adquiridos en su formación profesional sin perder de vista que los dicentes son sujetos en constante transformación y que están inmersos en un conjunto de interrelaciones sociales que los determinan. Para lograr este proceso de autorreflexión, un punto central de análisis y discusión es el umbral de relación que existe entre las estrategias de enseñanza aplicadas y las estrategias de aprendizaje que poseen los estudiantes. Otros aspectos, no menos importantes, son la identificación de los principios metodológicos que sustentan la práctica pedagógica y la implementación de innovaciones educativas para la atención de esta población estudiantil que demanda cada día más diversificar los espacios de intervención pedagógica. 


\section{REFERENCIAS}

Alcudia, R. Marisa, C., Gavilán, P., Sacristán, G., Giné, N., López, F., Montón, M. J., Onrubia, J., Pérez, C., Santamartí, N., Sentís, F., Tirado, V. y Viera, A. (2000) Atención a la diversidad. España: Graó.

Coll, C., Martín, E., Mauri, T., Miras, M., Onrubia, J., Solé, I. y Zabala, A. (1994) El constructivismo en el aula. España: Graó.

Gutiérrez, F. (1991) La mediación pedagógica. Apuntes para una educación a distancia alternativa. San José: RNTC.

Jiménez, R. (2000) Análisis Crítico del Currículo. Seminario Internacional. Educación para la Paz en el contexto rural. Heredia: Universidad Nacional.

Meléndez, L. (2002) La inclusión escolar del alumno con discapacidad intelectual. Bogotá: Creamos alternativas GLARP-IIPP.

Sánchez, M., Flores, L. y Céspedes, R. (2001) Comunicación y lengua escrita: transitando entre el contexto social el texto escrito. San José: Ministerio de Educación Pública OIM USAID Universidad Nacional.

Tébar, B. (2003) El perfil de profesor mediador. Madrid: Aula XXI. 